\title{
On Market Completions Approach to Option Pricing
}

\author{
Ilia Vasileva ${ }^{\mathrm{a}}$, Alexander Melnikov ${ }^{\mathrm{b}}$ \\ University of Alberta, Edmonton, AB, Canada \\ aWoS ResearcherID AAR-3773-2021 \\ bhttps://orcid.org/0000-0001-6261-0806 \\ ${ }^{b}$ Web of Science ResearcherID P-4620-2017
}

\begin{abstract}
Option pricing is one of the most important problems of contemporary quantitative finance. It can be solved in complete markets with non-arbitrage option price being uniquely determined via averaging with respect to a unique risk-neutral measure. In incomplete markets, an adequate option pricing is achieved by determining an interval of non-arbitrage option prices as a region of negotiation between seller and buyer of the option. End points of this interval characterise the minimum and maximum average of discounted pay-off function over the set of equivalent risk-neutral measures. By estimating these end points, one constructs super hedging strategies providing a risk-management in such contracts. The current paper analyses an interesting approach to this pricing problem, which consists of introducing the necessary amount of auxiliary assets such that the market becomes complete with option price uniquely determined. One can estimate the interval of non-arbitrage prices by taking minimal and maximal price values from various numbers calculated with the help of different completions. It is a dual characterisation of option prices in incomplete markets, and it is described here in detail for the multivariate diffusion market model. Besides that, the paper discusses how this method can be exploited in optimal investment and partial hedging problems.
\end{abstract}

Keywords: option pricing; complete markets; incomplete markets; non-arbitrage prices; hedging strategies; risk-management

For citation: Vasilev, I., Melnikov, A. (2021). On market completions approach to option pricing. Review of Business and Economics Studies, 9(3),77-93. DOI: 10.26794/2308-944X-2021-9-3-77-93

ОРИГИНАЛЬНАЯ СТАТЬЯ

\section{О методе рыночных пополнений в задачах оценки стоимости опционов}

\author{
Илья Васильев, Александр Мельников \\ Университет Альберты, Эдмонтон, АВ, Канада
}

\begin{abstract}
АННОТАЦИЯ
Задача оценки стоимости опционов является одной из самых важных в области современных математических финансов. В случае полного рынка стоимость опциона, исключающая арбитраж, может быть определена единственным образом посредством усреднения по единственной риск-нейтральной мере. Для неполного рынка, однако, риск-нейтральная мера не уникальна и возможно оценить стоимость опциона в виде интервала цен, не допускающих арбитраж, которые были бы приемлемы как для продавца, так и для покупателя контракта. Граничные точки такого интервала характеризуют минимальную и максимальную стоимость, на множестве эквивалентных риск-нейтральных мер данного рынка, а также средние стоимости дисконтированной функции выплаты опциона. Зная границы полученного интервала, в целях риск-менеджмента, инвестор формирует супер-хеджирующие стратегии. В настоящей работе приводится оригинальный подход к решению проблемы оценки границ безарбитражной стоимости опциона на неполном рынке. Суть подхода заключается в добавлении необходимого числа вспомогательных активов с целью получения полного рынка, на котором задача имеет единственное решение. Рассматривая все-
\end{abstract}

(C) Ilia Vasilev, Alexander Melnikov, 2021 
возможные пополнения, возможно также оценить минимальную и максимальную стоимости опционов на неполном рынке и получить интервал безарбитражных цен. Такое описание является дуальной характеристикой интервала стоимости опциона на неполном рынке. Авторы детально рассмотрели применение данного подхода к многомерной диффузионной модели рынка и обсудили возможность применения данного подхода при решении задач неполного хеджирования и оптимального инвестирования.

Ключевые слова: ценообразование опционов; полные рынки; неполные рынки; неарбитражные цены; стратегии хеджирования; управление рисками

\section{Introduction}

The problem of option pricing remains one of the most attractive and valuable problems. Mathematically, this problem admits a perfect solution if the market is complete, i.e., every contingent claim is attainable in the class of self-financing strategies or, equivalently, only one risk-neutral measure exists. Averaging over such a measure leads to a unique option price, called fair price in such a market. In an incomplete market, where non-attainable contingent claims exist, the situation is much more complicated because there are infinitely many risk-neutral measures. Averaging given discounted contingent claim over each such measure, one can get the whole interval of non-arbitrage option prices in contrast to one price in a complete market. So, in incomplete markets, to solve the option pricing problem, one needs to calculate the end points of this interval or provide their estimates.

In the present paper, we describe a fruitful method of solving the problem mentioned above. The leading idea of the proposed method is to transform the initial incomplete market model in such a way to make it complete and, hence, make it possible to calculate the unique price for a given contingent claim. Further, considering all possible transformations of the initial model, we get a set of non-arbitrage option prices similar to the set that existed in the classical approach. These findings lead to the dual characterisation of this set via minimal and maximal values as lower and upper option prices. Such a method of market completions was independently proposed for different incomplete market models: Karatzas (1997) for multivariate diffusion models, Melnikov and Feoktistov, (2001) and also Appendix 3 of Melnikov (1999) - for multinomial markets. The approach also works for pricing American options too (see, Guilan, 1999). Since that time, option pricing theory was tremendously developed in different aspects, including imperfect hedging, utility-indifference pricing, etc. It is pretty natural to expand the range of its applications.

We demonstrate that instead of using a set of equivalent local risk-neutral measures as a parameter for fair price interval estimation, an agent can work with an easier-to-interpret set of possible completion assets. For obvious reasons, this approach opens a way to nice flexibility of auxiliary assets and greater practical application as one can potentially find necessary assets to complete the market.

The method of market completions can mainly be used in two different ways. The first approach consists in the estimation of the price intervals. As there is a set of possible orthogonal completions available, one may aim at the estimation of the intervals of optimal prices that can be uniquely calculated in complete markets. The second approach is to pick particular completion. This idea is similar to choosing a specific measure of risks such as Esscher measure or Minimal Relative Entropy measure (see, for example, Miyahara, 1995). The second approach allows us to be more specific regarding assets required for the market to be complete. In some cases, it might be even possible to reverse-engineer such auxiliary assets, for instance, with the help of the BSDE technique (see Kobylanski, 2000).

In addition to option pricing problems, investors are also interested in finding an optimal strategy in incomplete market, often with some constraints. So, it is natural to look towards applying the proposed dual characterisation for these types of problems. There is a well-developed study in the area of partial hedging in complete markets. In Föllmer and Leukert (1999) and Spivak and Cvitanic (1999), authors considered quantile hedging, or maximisation of the probability of successful perfect-hedging, in Föllmer and Leukert (2000), authors also investigated shortfall minimi- 
sation in line with its utility-weighted value minimisation. These articles lay a foundation of partial hedging with the help of NeymanPearson lemma and Convex optimisation methods. Since recently, risk exposure is measured with the help of special measures widely used by market participants: Value-at-Risk (VaR) and Conditional Value-at-Risk (CVaR). The latter one is better known as Expected Shortfall (ES) and was recommended in 2016 in The Market Risk Framework of Basel III - an international regulatory accord. These measures spark a particular interest in their application in the optimal partial-hedging problem. Melnikov \& Smirnov (2012) show that it is still possible to apply Neyman-Pearson lemma to CVaR optimisation. Recent papers Cong et al. (2014), Li and Xu (2013), Capinski (2014), and Godin (2015) demonstrate a growing interest in CVaR optimisation. We will demonstrate how the method of market completions becoming a useful tool when solving this type of problems on an incomplete market.

The rest of the paper is structured as follows: Section 2 provides necessary details regarding the model under consideration. With the understanding of the reasons for market structural incompleteness, we move on to the central part of the paper - introducing the Method of Market Completions, which is discussed in Section 3 in line with its comparison to classical methodologies risk-neutral price interval estimation on the incomplete market. Section 4 elaborates on connections between market completions and some alternative methods used for handling market incompleteness. Finally, we briefly cover potential further steps towards solving famous partial hedging problems on the incomplete market in Section 5 and conclude the paper in Section 6.

\section{Multivariate Diffusion Market Model}

To demonstrate results that follow, we will work with the Standard Multidimensional Market Model, which is defined as $(B, S)=\left(B_{t}, S_{t}^{1}, \ldots, S_{t}^{n}\right)_{t \leq T}$, where $\left(B_{t}\right)_{t \leq T}$ represents the value process of a risk-free asset that is usually assumed to be a bank account and $S_{t}=\left(S_{t}^{1}, \ldots, S_{t}^{n}\right)_{t \leq T}$ is a $n$-dimensional vector process that describes the prices of $n$ risky assets:

$$
\begin{array}{r}
d B_{t}=B_{t} r_{t} d t, \quad B_{0}=1 \\
d S_{t}^{i}=S_{t}^{i}\left(\mu_{t}^{i} d t+\sum_{j=1}^{k} \sigma_{t}^{i j} d W_{t}^{j}\right)
\end{array}
$$

We will also call $\Sigma_{t}=\left\{\sigma_{t}^{i j}\right\}_{i, j}$ a volatility matrix of this model. Note that elements of a $k$-dimensional vector $W=\left(W^{1}, \ldots, W^{k}\right)$ are independent standard Brownian motions. In general, one can define a multidimensional market model so that each risky asset price is governed by its own separate Brownian motions that are mutually correlated. However, it was shown, for example, in Dhaene et al. (2013), that both mentioned models are equivalent. Further in this paper, we will use the model with independent "underlying” Brownian motions for illustration.

Let us call the $(\mathcal{F})_{t \leq T}$-measurable process $\pi=\left(\beta_{t}, \pi_{t}^{1}, \ldots, \pi_{t}^{n}\right)_{t \leq T}$ a portfolio (strategy). This process would reflect amounts of corresponding assets possessed by an investor. Obviously, the capital or value of such a portfolio can be described as

$$
V_{t}^{\pi}=\beta_{t} B_{t}+\sum_{i=1}^{n} \pi_{t}^{i} S_{t}^{i}
$$

Note that not all strategies would be appropriate for the investor. Typically, the agent on the market has an initial budget $x$, and the natural constraint is that strategy value should not fall below some threshold at any moment $t$ while strategy is in action. To accommodate this condition, denote the class of admissible portfolios with initial capital $x$ as

$\mathcal{A}(x)=\left\{\pi: V_{0}^{\pi}=x, \exists K(\pi) \geq 0\right.$ s.t. $\left.V_{t}^{\pi} \geq-K, \forall t \leq T\right\}$.

For simplicity, we might consider $K=0$, meaning that the investor does not want his portfolio to have negative value at any moment until the maturity of the strategy.

Admissible strategy $\pi$ is called self-financing if the following conditions hold:

$$
\begin{gathered}
\int_{0}^{T} \sum_{i=1}^{n}\left(\left|\pi_{t}^{i} \mu_{t}^{i}\right|+\left(\pi_{t}^{i}\right)^{2} \sum_{j=1}^{k}\left(\sigma_{t}^{i j}\right)^{2}\right) d t<\infty \\
V_{t}^{\pi}=V_{0}^{\pi}+\sum_{i=1}^{n} \int_{0}^{t} \pi_{s}^{i} d S_{s}^{i} .
\end{gathered}
$$


In other words, strategy is called self-financing if its capital changes only due to changes in asset prices without additional injections or extractions of capital by the investor. We will denote the class of self-financing strategies with initial capital $x$ as $S F(x)$.

Definition 0.1: Model is called arbitrage-free if there is no strategy $\pi \in S F(x)$ such that it has zero initial cost of investment and leads to non-zero profit at maturity with positive probability:

$$
V_{0}^{\pi}=0, \quad P\left(V_{T}^{\pi}>0\right)>0 .
$$

It is well known that the market model is arbitrage-free if and only if there exists an equivalent martingale measure. It was shown in Karatzas and Shreve (2000) that for the Standard Multidimensional Market Model (1), the no-arbitrage condition could be summarised in the following proposition.

Proposition 0.1: If there exists a $\left(\mathcal{F}_{\mathrm{t}}\right)_{\mathrm{t} \leq \mathrm{T}}-$ progressively measurable process $\theta=\left(\theta_{t}^{1}, \ldots, \theta_{t}^{k}\right)_{t \leq T}$ that satisfies

$$
\sum_{j=1}^{k} \sigma_{t}^{i j} \theta_{t}^{j}=\mu_{t}^{i}-r, \quad i=1, \ldots, n, \quad P-a . s .
$$

and

$$
\mathbb{E}\left[\exp \left(\frac{1}{2} \int_{0}^{T} \sum_{j=1}^{k}\left(\theta_{t}^{j}\right)^{2} d t\right)\right]<\infty
$$

then the $(B, S)$ the market is arbitrage free.

In other words, the market is arbitrage-free if system (4) has the solution.

Remark: The inverse Proposition 0.1 is, in general, not true. Condition (4) should hold. However, Novikov condition (5) is sufficient but not a necessary one for uniform integrability of Girsanov exponent and, consequently, for equivalence of corresponding risk-neutral measure.

Remark: Solution to the system (4): $\theta_{t}$ is, actually, the one to use for the famous Girsanov theorem to switch to equivalent risk-neutral measure under which discounted risky assets in the model (1) become martingales.

Remark: Condition (4) can be equivalently written in a vector form:

$$
\Sigma_{t} \theta_{t}=\mu_{t}-r
$$

where $\theta_{t} \in \mathbb{R}^{k} ; \mu_{t}, \boldsymbol{r} \in \mathbb{R}^{n} \forall t \in[0, T]$.
Denoting $\left\|\sigma_{t}^{i}\right\|=\sqrt{\sum_{j=1}^{k}\left(\sigma_{t}^{i j}\right)^{2}}$, condition (5) can
also be written as:

$$
\mathbb{E}\left[\exp \left(\frac{1}{2} \int_{0}^{T}\left\|\sigma_{t}^{i}\right\|^{2} d t\right)\right]<\infty
$$

\section{Market Completeness}

Definition 0.2: (Market completeness) The market is called complete if for any $\mathcal{F}_{\mathrm{T}}-$ measurable payment function $H=H_{T}(\omega) \geq 0$, such that $\mathbb{E}[\mathrm{H}]<\infty$ there exists a strategy $\pi \in S F(x)$ such that $\mathbb{P}$-a.s.

$$
V_{T}^{\pi}(x)=H \text {. }
$$

Generally speaking, market incompleteness means that sigma algebra $\mathcal{F}_{T}^{S}$ generated by risky assets is smaller than $\mathcal{F}$ on which contingent claims are defined, namely, $\mathcal{F}_{T}^{S} \subset \mathcal{F}$. There might be different reasons for market incompleteness, including, but not limited to:

1. Structural: There are more sources of risks on the market than tradeable assets available. In such a case, it is natural to define sigma algebra for claims as the one generated by underlying sources of risk. In the case of model (1), it would be $\mathcal{F}_{T}^{W}$.

2. Informational: Some investors may have more information regarding the asset price dynamics on the market than others. Typical cases of Large investor were described in Eyraud-Loisel (2019); Follmer and Schweizer (1991).

3. Due to complex parameters or restrictions: When parameters of the model become stochastic values (stochastic volatility, stochastic drift, etc.) which are not observable explicitly on the market.

In this paper, we will focus on the structural incompleteness of the market. Condition for such incompleteness in case of (1) was obtained in Karatzas and Shreve (2000) and Dhaene et al. (2013). We summarise them in the following theorem.

Theorem 1: Standard financial market $\mathcal{M}$ is complete if and only if a number of available stocks $n=k$, where $k$ is a dimension of underlying Brownian motion.

Consequently, to have a complete market, we need to have a proper, non-degenerate volatility 
matrix $\Sigma_{t}$ with $n=k$. As market completeness means the existence of a unique martingale measure $\mathbb{P}^{*}$, the market is complete if system (4) possesses the unique solution $\theta_{t} \in \mathbb{R}^{k}$. Girsanov exponential for transition to that unique martingale measure in the multidimensional case will be written in the following form:

$$
\frac{d P^{*}}{d P}=\exp \left\{-\sum_{i=1}^{n} \int_{0}^{T} \theta_{t}^{i} d W_{t}^{i}-\frac{1}{2} \sum_{i=1}^{n} \int_{0}^{T}\left(\theta_{t}^{i}\right)^{2} d t\right\} .
$$

\section{Completions of Diffusion Model and Option Pricing}

We now move on and introduce the method of market completions which is the main focus of the present paper. First, we formalise the notion of market completion.

As we already noted, markets, in reality, are barely complete, so it is reasonable to find a way to handle market incompleteness. In the previous chapter, we showed that, when speaking about structural incompleteness, such incompleteness for Standard Multidimensional Diffusion market model demonstrated through the volatility matrix which rank is not full. Or, roughly speaking, when the volatility matrix for tradeable assets has a rectangular shape with more columns (sources of risks represented by independent Brownian motions) than rows (risky assets).

In other words, to obtain a complete market that would correspond to the existing incomplete one, it is reasonable to add more "rows" into the volatility matrix under consideration. This idea forms a foundation of the method of market completions.

Obviously, "completing" assets should be independent of existing ones and among each other to solve the issue of a non-full rank volatility matrix. Adding them, we obtain a "proper" volatility matrix that corresponds to some complete market where known and well-developed methods can be applied.

\section{Definitions of the Method of Market Completions}

Assume the canonical market model (1) with $n$ risky assets for which $n<k$. As always, asset price dynamics is defined on measure space $(\Omega, \mathcal{F}, P)$ equipped with filtration $\mathbb{F}$ generated by $k$-dimensional Brownian motion. We will call assets that form this incomplete model primary assets or existing assets.

Denote $S^{c}$ a $(k-n)$-dimensional $\left(\mathcal{F}_{t}\right)_{t \leq T}-$ adapted process $S^{c}=\left(S_{t}^{n+1}, \ldots, S_{t}^{k}\right)_{t \leq T}$ with the same structure as primary assets:

$$
d S_{t}^{i}=S_{t}^{i}\left(\mu_{t}^{i} d t+\sum_{j=1}^{k} \sigma_{t}^{i j} d W_{t}^{j}\right), \quad i=n+1, \ldots, k .
$$

With the help of newly introduced assets, we can "fix" initially rectangular volatility matrix for a set of existing risky assets $\sigma$ :

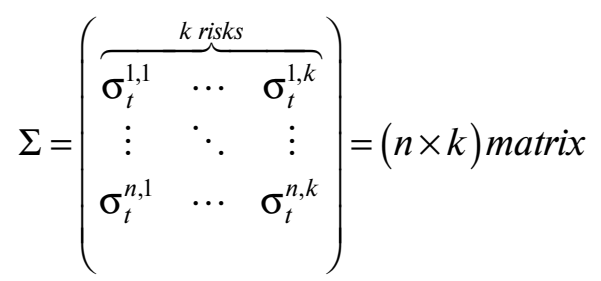

by adding $k-n$ auxiliary assets introduced:

$$
\tilde{\Sigma}=\left(\begin{array}{ccc}
\sigma_{t}^{1,1} & \cdots & \sigma_{t}^{1, k} \\
\vdots & \ddots & \vdots \\
\sigma_{t}^{n, 1} & \cdots & \sigma_{t}^{n, k} \\
& & \\
\sigma_{t}^{n+1,1} & \cdots & \sigma_{t}^{n+1, k} \\
\vdots & \ddots & \vdots \\
\sigma_{t}^{k, 1} & \cdots & \sigma_{t}^{k, k}
\end{array}\right)=(k \times k) \text { matrix }
$$

Which helps us to arrive at a properly shaped volatility matrix $\tilde{\Sigma}$.

Definition 0.1: The $(\mathrm{k}-\mathrm{n})$-dimensional $\left(\mathcal{F}_{\mathrm{t}}\right)_{\mathrm{t} \leq \mathrm{T}}$ - adapted process $\mathrm{S}^{\mathrm{c}}=\left(\mathrm{S}_{\mathrm{t}}^{\mathrm{n}+1}, \ldots, \mathrm{S}_{\mathrm{t}}^{\mathrm{k}}\right)_{\mathrm{t} \leq \mathrm{T}}$ is called a completion for the $(B, S)$ market if the resulting volatility matrix $\tilde{\Sigma}$ has full rank for all $\mathrm{t} \leq \mathrm{T}$.

Definition 0.2: A completion $\overline{\mathrm{S}}^{\mathrm{c}}=\left(\overline{\mathrm{S}}^{\mathrm{n}+1}, \ldots, \overline{\mathrm{S}}^{\mathrm{k}}\right)$ is called orthogonal if it satisfies:

$$
S_{t}^{i}, \bar{S}_{t}^{j}=0, \text { for all } i=1, \ldots, n ; j=n+1, \ldots, k ; t \in[0, T]
$$

and

$$
\bar{S}_{t}^{i}, \bar{S}_{t}^{j}=0, \text { for all } i, j=n+1, \ldots, k ; t \in[0, T]
$$

Remark: Operation $\langle\cdot, \cdot\rangle$ is taken from the standard martingale theory and represents the 
quadratic covariation of martingale parts of the processes.

Further in this paper, the set of orthogonal completions will be denoted as $\mathcal{C}^{\text {ort }}$. We demonstrate that any market completion can be transformed into an orthogonal form.

Lemma 1: For any completion $\mathrm{S}^{\mathrm{c}} \in \mathcal{C}$ of the $(\mathrm{B}, \mathrm{S})$ market, it is possible to find an orthogonal completion $\overline{\mathrm{S}}^{\mathrm{c}} \in \mathcal{C}^{\text {ort }}$.

\section{Proof}

It is enough to show that one can always construct orthogonal completion from nonorthogonal assets. It can be accomplished, for example, with the help of a famous Gram-Schmidt method. Our goal is to construct a process $\bar{S}^{c}=\left(\bar{S}^{n+1}, \ldots, \bar{S}^{k}\right)$ that satisfies the definition above.

To do it, we first define the stochastic logarithm $H^{i}=\left(H_{t}^{i}\right)_{t \leq T}$ :

$$
d H_{t}^{i}=\frac{d S_{t}^{i}}{S_{t}^{i}}=\mu_{t}^{i} d t+\sum_{j=1}^{k} \sigma_{t}^{i j} d W_{t}^{j}
$$

Considering that $i \neq j$, if $H_{t}^{i}, H_{t}^{j}=0$ for all $t \in[0, T]$ then $S_{t}^{i}, S_{t}^{j}$. On the other hand, if rowvectors $\sigma_{t}^{i}$ and $\sigma_{t}^{j}$ of volatility matrix are orthogonal for $i \neq j$ for all $t \in[0, T]$, then

$$
\left\langle H_{t}^{i}, H_{t}^{j}\right\rangle=\left\langle\begin{array}{c}
H_{0}^{i}+\int_{0}^{t} \mu_{s}^{i} d s+\sum_{l=1}^{k} \int_{0}^{t} \sigma^{i l} d W_{s}^{l}, H_{0}^{j}+ \\
+\int_{0}^{t} \mu_{s}^{j} d s+\sum_{l=1}^{k} \int_{0}^{t} \sigma^{j l} d W_{s}^{l} \\
=\int_{0}^{t} \sum_{l=1}^{k} \sigma_{s}^{i l} \sigma_{s}^{j l} d s
\end{array}\right\rangle=
$$

Consequently, to complete the proof, it is enough to show how to construct orthogonal row-vectors $\bar{\sigma}_{t}^{j}$ and it would imply orthogonality of assets.

To construct such vectors, we will use the Gram-Schmidt method of orthogonalisation for $\sigma_{t}^{i}, i=1, \ldots, k$ :

$$
\begin{array}{r}
\bar{\sigma}_{t}^{1}=\sigma_{t}^{1}, \\
\bar{\sigma}_{t}^{i}=\sigma_{t}^{i}-\sum_{j=1}^{i-1} \alpha_{t}^{i j} \bar{\sigma}_{t}^{j},
\end{array}
$$

f o r $\quad i=2, \ldots, k \quad$ w i t h $\quad \alpha_{t}^{i j}=\frac{\sigma_{t}^{i}, \bar{\sigma}_{t}^{j}}{\bar{\sigma}_{t}^{j}, \bar{\sigma}_{t}^{j}} \quad$ f o r $i, j=2, \ldots, k ; j<i$. It is easy to see that obtained vectors are indeed orthogonal.

Let us also obtain the assets for completion. Defining $\bar{H}^{i}=\left(\bar{H}_{t}^{i}\right)_{t \leq T}$ for $i=k+1, \ldots, n$ as

$$
\begin{array}{r}
d \bar{H}^{i}=\bar{\mu}_{t}^{i}+\sum_{l=1}^{n} \bar{\sigma}_{t}^{i l} d W_{t}^{j}, \\
\bar{\mu}_{t}^{1}=\mu_{t}^{1}, \\
\bar{\mu}_{t}^{i}=\mu_{t}^{i}-\sum_{j=1}^{i-1} \alpha_{t}^{i j} \bar{\mu}_{t}^{j},
\end{array}
$$

with

for $i=2, \ldots, n$. Final completion assets can be obtained from:

$$
d \bar{S}_{t}^{j}=\bar{S}_{t}^{j} d \bar{H}_{t}^{j}, j \in \overline{k+1, n}
$$

Remark: Orthogonalisation of drift terms for assets in the proof of lemma above plays a rather technical role. In such a form, one would get a much simpler solution for the (4).

\section{Working with the Set of Orthogonal Completions Instead of ELMM}

Let us now demonstrate that working with the set of possible orthogonal completions would be equivalent to working with the set of equivalent local martingale measures (ELMM). As a reminder, an equivalent probability measure is called equivalent (local) martingale measure if discounted risky asset price under such measure is a (local) martingale. We will demonstrate this in case of the problem of estimation of risk-neutral price interval for an initially incomplete market model.

It is well known that in incomplete markets, there are infinitely many ELMMs. Consequently, the risk-neutral price is not unique, and it is more reasonable to speak about the interval of initial fair prices. From the classical martingale approach, it is known that this interval could be described as:

$$
\left(\inf _{\tilde{P} \in \mathcal{M}} E^{\tilde{P}}\left[\frac{f_{T}}{B_{T}}\right], \sup _{\tilde{P} \in \mathcal{M}} E^{\tilde{P}}\left[\frac{f_{T}}{B_{T}}\right]\right)
$$

where $f_{T}$-contingent claim maturing at time $T$ and $\mathcal{M}$ - set of all ELMMs.

We will demonstrate that fair price interval boundaries obtained with the help of the method of Market Completions coincide with ones from the 
classical approach. It is straightforward that by "completing" our $(B, S)$ the market we arrive at volatility matrix $\tilde{\Sigma}$ and force the system (4), or, in this case

$$
\tilde{\Sigma}_{t} \theta_{t}=\mu_{t}-r, P-a . s .
$$

to have a unique solution $\theta$.

For this completed market model, there should exist unique equivalent local martingale measure, parametrised with the help of solution $\left\{\theta_{t}\right\}_{t \geq 0}$. As each "completed" volatility matrix corresponds to particular market completion, there is a one-to-one correspondence between the set of ELMM for the initial incomplete model and a set of orthogonal completions.

\section{Lemma 2:}

A. Each completion $S^{c}$ uniquely defines a single ELMM in the incomplete market. Moreover, for the equivalent orthogonal complete market (obtained using the method of Lemma 1), such local martingale measure will be the same.

B. Each ELMM $\tilde{P}$ in the incomplete market ( $\tilde{P} \in \mathcal{M}$ ) will be a unique ELMM in the associated completed market model. Therefore, the set $\mathcal{M}$ of ELMMs in the incomplete market is equivalent to the set $\mathcal{M}^{c}$ of unique ELMMs corresponding to each completion of the market.

This beautiful fact allows us to switch analysis from a very abstract class of Equivalent Martingale measures to a class of "completing" assets. The latter is much easier to interpret and also impose different restrictions such as maximal asset volatility or no short selling on the market. For now, let us focus on fair price calculation.

Theorem 2: In the incomplete $(\mathrm{B}, \mathrm{S})$ market, assume that $\mathrm{r}=0$ and let $\overline{\mathrm{M}}_{\mathrm{t}}^{\mathrm{i}}$ and $\overline{\mathrm{\sigma}}_{t}^{i}=\left(\overline{\mathrm{\sigma}}_{t}^{i 1}, \ldots, \bar{\sigma}_{t}^{i k}\right)$ be as defined in the proof of Lemma 1 for $\mathrm{i}=1, \ldots, \mathrm{n}$ . Let also $\overline{\mathrm{W}}$ be a standard $\mathrm{k}$-dimensional Brownian motion, with the first $\mathrm{n}$ elements given by

$$
\bar{W}_{t}^{i}=\frac{1}{\bar{\sigma}_{t}^{i}} \sum_{j=1}^{k}{\overline{\sigma_{t}}}^{i j} W_{t}^{j}
$$

for $i=1, \ldots, n, t \in[0, T]$, where $\bar{\sigma}_{t}^{i}=\sqrt{\sum_{j=1}^{k}\left(\bar{\sigma}_{t}^{i j}\right)^{2}}$. Then the upper hedging price can be expressed as

$$
\begin{gathered}
C^{*}\left(f_{T}, P\right)= \\
=\sup _{\frac{\bar{\mu}^{i}}{\bar{\sigma}_{t}^{i}}, i=n+1, \ldots, k} E^{P}\left[\exp \left\{\begin{array}{c}
-\sum_{i=1}^{k} \int_{0}^{T} \frac{\bar{\mu}^{i}}{\bar{\sigma}_{t}^{i}} d \bar{W}_{t}- \\
-\frac{1}{2} \sum_{i=1}^{k} \int_{0}^{T}\left(\frac{\bar{\mu}^{i}}{\bar{\sigma}_{t}^{i}}\right)^{2} d t
\end{array}\right\} f_{T}(\bar{W})\right] .
\end{gathered}
$$

\section{Change of Numeraire}

In line with the Equivalent Martingale Measure approach, it is also worth mentioning the socalled change of numeraire pricing approach. Its connection to the method of market completions was described in Guilan (1999). We provide the main steps below for informational purposes and to complete an overview of the method of Market Completions in application to pricing problem.

According to this approach, instead of trying to "re-weight" the probability of events by choosing some risk-neutral measure, one is searching for a special portfolio that could be used as discounting factor instead of the classical bank account. However, the choice criteria for such discounting portfolio stays the same - discounted strategy prices should be martingales.

More formally, the main goal is to find a portfolio, which value process $X_{t}$ is a strictly positive, continuous Ito process such that:

$$
d X_{t}=X_{t}\left(r_{t} d t+\pi_{t}^{*} \sigma_{t}\left(d W_{t}+u_{t} d t\right)\right) \text {. }
$$

Remark: Here, we will intentionally use notation $u$ instead of $\theta$ just to distinguish approaches. However, they both represent the same idea of the price of the risk.

We want to use this portfolio as numeraire, such that risk-premiums with respect to this numeraire are constrained to be equal 0 . In other words, the price process, discounted by a mentioned portfolio, will be local martingale w.r.t. "objective" probability $P$.

Theorem 3: Let $\alpha_{t}=\left(\sigma_{t} \sigma_{t}^{T}\right)^{-1}\left(\mu_{t}-r_{t} 1\right)$, i.e., $u_{t}=\sigma_{t}^{T} \cdot \alpha_{t}$. Consider the self-financing strategy $\pi_{t}=\left(\alpha_{t}^{i}\right)_{i=1}^{n}$ in the risky assets. Denote by $\mathrm{M}_{\mathrm{t}}$ the present value of this admissible strategy. Then $\mathrm{M}_{\mathrm{t}}$ satisfies $S D E$ :

$$
\begin{gathered}
d M_{t}=M_{t}\left(r_{t} d t+\left(u_{t}\right)^{T}\left(d W_{t}+u_{t} d t\right)\right)= \\
=M_{t}\left(r_{t} d t+\left\|u_{t}\right\|^{2} d t+\left(u_{t}\right)^{T} d W_{t}\right)
\end{gathered}
$$

In the market with $M_{t}$ as numeraire, investors are risk-neutral. M-price process $S_{t}^{M}=\frac{S_{t}}{M_{t}}$ of any asset $S_{t}$ is a local martingale. We refer to it as a market numeraire.

Proposition 0.1: If $\mathrm{m}$ is a strategy that corresponds to $\mathrm{M}_{\mathrm{t}}$, then: 
- $m$ maximises the expected logarithm of terminal wealth

- $m$ is unique even in an incomplete market

- $m$ maximises the expected growth rate.

Details about mentioned properties can be found in Bajeux-Besnainou and Portait (1997).

Price of European contingent claim $f_{T}$ on the complete market, according to market numeraire approach could be found as:

$$
V_{0}=\mathbb{E}^{P}\left(\frac{f_{T}}{M_{T}}\right)
$$

When one is working with the incomplete market case, it is obvious that there are several risk-neutral prices, as we can find several $\alpha_{t}$ that fit conditions of Theorem 2. Let us now apply the market completions approach and show that it can estimate option price boundaries on an incomplete market.

Let us consider some market completion $S^{c}$. Then coefficients of these fictitious assets satisfy

$$
\operatorname{det}(\sigma(\rho))=\operatorname{det}\left(\begin{array}{l}
\sigma_{t} \\
\rho_{t}
\end{array}\right) \neq 0 \text { and } u(\rho, a, t)=\left(\begin{array}{c}
\sigma_{t} \\
\rho_{t}
\end{array}\right)^{-1}\left(\begin{array}{c}
b_{t}-r_{t} I_{n} \\
a_{t}-r_{t} I_{k-n}
\end{array}\right)
$$

with

$$
\int_{0}^{T}\|u(\rho, a, t)\|^{2} d t<\infty, \quad P-a . s
$$

On the completed market, one can define market numeraire as in (11):

$$
d M(\rho, a, t)=M(\rho, a, t)\left(r_{t} d t+\|u(\rho, a, t)\|^{2} d t+(u(\rho, a, t))^{T} d W_{t}\right)
$$

In the completed market, we have the fair price of CC $f_{T}$ calculated similarly to (12):

$$
V_{0}(\rho, a)=\mathbb{E}^{P}\left(\frac{f_{T}}{M(\rho, a, T)}\right)
$$

Let

$$
V_{1}(\rho)=\inf _{a \in \mathcal{D}_{\rho}} V_{0}(\rho, a), \quad V_{2}(\rho)=\sup _{a \in \mathcal{D}_{\rho}} V_{0}(\rho, a)
$$

with $\mathcal{D}_{\rho}=\left\{a: \mathbb{R}^{k-n}\right.$ valued progressively measurable processes such that $\int_{0}^{T}\|u(\rho, a, t)\|^{2} d t<\infty$ a.s. $\}$.

According to Guilan (1999), the following proposition holds.

Proposition 0.1: $V_{1}(\rho)$ and $V_{2}(\rho)$ are independent of $\rho$.

Proposition 0.1 serves as another proof that it is enough to work with orthogonal completions only. Let us pick the orthogonal completion $\sigma \rho^{T}=0, \rho \rho^{T}=I$. For such $\rho$ and $a \in \mathcal{D}_{\rho}$ :

$$
u(\rho, a)=\left(\begin{array}{c}
\sigma \\
\rho
\end{array}\right)^{-1}\left(\begin{array}{c}
\mu_{t}-r I_{n} \\
a_{t}-r I_{k-n}
\end{array}\right)=\sigma^{T}\left(\sigma \sigma^{T}\right)^{-1}\left(\mu-r I_{n}\right)+\rho^{T}\left(a-r I_{k-n}\right)=u+\psi=u_{\psi} .
$$

And this $u_{\psi}$ would be used for the construction of market numeraire. Also, it follows that and $\sigma \psi=0$,

$a=\rho \psi+r I_{k-n}$

It means that the "non-arbitrage" vector $u_{\psi}$ on the completed market can be decomposed into $u$ from incomplete source market and $\psi$ which is completion dependent. If we define class 
$K(\sigma)=\left\{\psi: \psi\right.$ is $\mathbb{R}^{k}$ - valued progressively measurable, $\sigma_{t} \psi_{t}=0, \forall t \in[0, T]$, a.s. and $\int_{0}^{T}\left\|\psi_{t}\right\|^{2} d t<\infty$, a.s. $\}$,

then this class will be a parameter space for fictitious completions of the incomplete market. For each $\psi \in K(\sigma)$ one can find a fair price in a completed market. It implies that option price boundaries will be

$$
J(t)=\sup _{\psi \in K(\sigma)} \mathbb{E}\left[M_{\psi}(t) \frac{f_{T}}{M_{\psi}(T)} \mid \mathcal{F}_{t}\right] \text { or } \inf _{\psi \in K(\sigma)} \mathbb{E}\left[M_{\psi}(t) \frac{f_{T}}{M_{\psi}(T)} \mid \mathcal{F}_{t}\right]
$$

Remembering results from Guilan (1999), it is possible to show that these price boundaries coincide with boundaries from the classical approach:

$$
V(t)=\sup _{\tilde{P} \in \mathcal{M}} \mathbb{E}^{\tilde{P}}\left[\mathrm{~B}_{\mathrm{t}} \frac{f_{T}}{\mathrm{~B}_{\mathrm{T}}} \mid \mathcal{F}_{t}\right] \text { or } \inf _{\tilde{P} \in \mathcal{M}} \mathbb{E}^{\tilde{P}}\left[\mathrm{~B}_{\mathrm{t}} \frac{f_{T}}{\mathrm{~B}_{\mathrm{T}}} \mid \mathcal{F}_{t}\right]
$$

In other words, it was also shown that $J(t)$ coincides with $V(t)$. For more details, we also encourage the reader to carefully read Guilan (1999) research.

\section{Completions in Context of Markov Factors, Dimension Reductions and Jumps}

Connection to Markov Factor Model
Denote Girsanov exponential (6) as $\bar{Z}_{t}=\frac{d P^{*}}{d P}$. It is known that this process is a solution for

$d \bar{Z}_{t}=\bar{Z}_{t} \bar{\theta}_{t} d W_{t}, \quad \bar{Z}_{0}=1$

and noting that $\bar{\theta}_{t}^{i}=\frac{\bar{\mu}_{t}^{i}}{\bar{\sigma}_{t}^{i}}$ from non-arbitrage condition $\Sigma_{t} \bar{\theta}_{t}=\bar{\mu}_{t}(r=0)$. Then equation (10) can be re-written in the following form:

$$
C^{*}\left(f_{T}, P\right)=\sup _{\theta} \mathbb{E}^{P}\left[Z_{T}(\theta) f_{T}(\bar{W})\right] .
$$

Moreover, as the first $k$ elements of the vector $\bar{\theta}$ are independent of the choice of completion and only depend on the correlations between existing assets, one can represent vector $\bar{\theta}$ as $\bar{\theta}_{t}=\bar{a}_{t}+\bar{c}_{t}, \bar{a}_{t}, \bar{c}_{t} \in \mathbb{R}^{n}$ where the first one contains elements of $\bar{\theta}_{t}$ calculated based on existing assets only. Namely:

$$
\begin{gathered}
\bar{a}=\left[\bar{\theta}_{t}^{1} \ldots \bar{\theta}_{t}^{k}, 0\right]^{T}, \\
\bar{c}=\left[0, \bar{\theta}_{t}^{k+1} \ldots \bar{\theta}_{t}^{n}\right]^{T} .
\end{gathered}
$$

In this case, the equation for Girsanov exponential can be re-written as

$$
d Z_{t}=Z_{t} \bar{a}_{t} d W_{t}+Z_{t} \bar{c}_{t} d W_{t}, \quad Z_{0}=1
$$

Hence, market completions can be connected to the Markov Factor Model:

$$
\begin{aligned}
& d S_{t}=D\left(S_{t}\right)\left(\mu\left(Y_{t}\right) d t+\sigma\left(Y_{t}\right) d W_{t}\right) \\
& d B_{t}=r B_{t} d t
\end{aligned}
$$

where $Y_{t}$ is $(k-n)$ dimensional factor process, which does not contain any price processes 


$$
d Y_{t}=\mu_{Y}\left(Y_{t}\right) d t+\sigma_{Y}\left(Y_{t}\right) d W_{t}
$$

$\mu_{Y}$ and $\sigma_{Y}$ are vector functions of appropriate dimensions. Or, more conveniently, to the Independent Factor Markov Model in which we assume that vector-valued Wiener process $W$ could be split as

$$
\mathrm{W}=\left(\begin{array}{l}
W^{S} \\
W^{Y}
\end{array}\right)
$$

such that $W^{S}$ is $n$-dimensional and corresponds to existing assets on the market and $W^{Y}$ is $(k-n)$ dimensional and corresponds to factors. In this setting, Markov Factor Model can be written as:

$$
\begin{aligned}
d S_{t} & =D\left(S_{t}\right)\left(\mu\left(Y_{t}\right) d t+\sigma\left(Y_{t}\right) d W_{t}^{S}\right), \\
d Y_{t} & =\mu_{Y}\left(Y_{t}\right) d t+\sigma_{Y}\left(Y_{t}\right) d W_{t}^{Y}, \\
d B_{t} & =r B_{t} d t .
\end{aligned}
$$

It is possible to show that split (15) is similar to what was demonstrated in (9) with existing assets on the incomplete market being assigned, in fact, to $\bar{W}^{i}$ for $i \in 1 . . n, t \in[0, T]$ which corresponds to $W^{S}$ and the rest of $\bar{W}^{i}$ being assigned to $W^{Y}$ as it only depends on $(k-n)$ dimensional Brownian motion.

Remark: To briefly demonstrate the idea of transformation completions notation into Markov Factor model one. Assume that we performed the transformation mentioned in (9) for the Standard Multidimensional Diffusion Market model. In this case, it is easy to see that the "completed" volatility matrix can be written as:

$$
\tilde{\Sigma}=\left(\begin{array}{cc}
L_{n \times n} & 0_{n \times(k-n)} \\
0_{(k-n) \times n} & D_{(k-n) \times(k-n)}
\end{array}\right)
$$

Where $\mathrm{L}_{\mathrm{n} \times \mathrm{n}}$ is a lower triangle matrix and $\mathrm{D}_{(\mathrm{k}-\mathrm{n}) \times(\mathrm{k}-\mathrm{n})}$ is a diagonal one. This leads to the natural split of vector $W$ into two parts. Without loss of generality, one might assume first $n$ elements of $W$ to be denoted as $\mathrm{W}^{\mathrm{S}} \in \mathbb{R}^{\mathrm{n}}$ and the last $(k-n)$ elements as $\mathrm{W}^{\mathrm{Y}} \in \mathbb{R}^{\mathrm{k}-\mathrm{n}}$.

\section{Dimension Reduction}

Another natural approach to transform the volatility matrix into a proper one would be to "trim" it. Or somehow "regroup" underlying Brownian motions in such a way that the reduced volatility matrix for them will have the proper shape. This idea was introduced by Zhang (2007).

For the introduced Standard Multidimensional Diffusion Market Model, dynamics of each risky asset price is governed by the sum of independent standard Brownian motions

$$
\begin{array}{r}
d S_{t}^{0}=S_{t}^{0} r_{t} d t, \quad S_{0}^{0}=1 \\
d S_{t}^{i}=S_{t}^{i}\left(\mu_{t}^{i} d t+\sum_{j=1}^{k} \sigma_{t}^{i j} d W_{t}^{j}\right), \quad i=1, \ldots, n .
\end{array}
$$

However, as already mentioned, it is possible to write down an equivalent market model which would be governed by $n$ correlated Brownian motions instead of $k$ independent ones (see, e.g., Dhaene et al., 2013):

$$
d B_{t}^{i}=\sum_{j=1}^{k} \frac{\sigma_{t}^{i j}}{\left\|\sigma_{t}^{i}\right\|} d W_{t}^{j}
$$

then

$$
d S_{t}^{i}=S_{t}^{i}\left(\mu_{t}^{i} d t+\left\|\sigma_{t}^{i}\right\| d B_{t}^{i}\right), \quad i=1, \ldots, n
$$

Obviously, obtained Brownian motions are not independent anymore, namely

$$
\begin{gathered}
d B_{t}^{i} d B_{t}^{j}=\rho_{t}^{i j} \\
\rho_{t}^{i l}=\frac{\sum_{j=1}^{k} \sigma_{t}^{i j} \sigma_{t}^{l j}}{\left\|\sigma_{t}^{i}\right\| \cdot\left\|\sigma_{t}^{l}\right\|} .
\end{gathered}
$$

In this model, we have $n$-dimensional Brownian motion vector with correlated components $B_{t}=\left(B_{t}^{1}, \ldots, B_{t}^{n}\right)$, the relationship between which can be described by matrix $\Psi_{t}=\left\{\rho_{t}^{i l}\right\}_{i, l=1 . . n}$. Notice that $\Psi_{t}$ is a non-singular, symmetric, and positive semi-definite. That implies the existence of matrix square-root $A_{t}$ :

$$
\Psi_{t}=A_{t} \cdot A_{t}^{T}, \quad A_{t}=\left\{a_{t}^{i j}\right\}_{i, j=1 . . n}
$$

Moreover, $\exists \tilde{W}_{t}^{1}, \ldots, \tilde{W}_{t}^{n}$ independent, such that:

$$
B_{t}^{i}=\sum_{j=1}^{n} \int_{0}^{t} a_{s}^{i j} d \tilde{W}_{s}^{j}
$$


As a result, risky assets can be presented in a form

$$
\begin{gathered}
d S_{t}^{i}=S_{t}^{i}\left(\mu_{t}^{i} d t+\sigma_{t}^{i} \sum_{j=1}^{n} a_{t}^{i j} d \tilde{W}_{t}^{j}\right), \quad i=1, \ldots, n . \\
\tilde{\sigma}_{t}=\rho A_{t}
\end{gathered}
$$

Where $\Xi_{t}$ is a diagonal matrix of $\sigma^{i}$ and matrix $A_{t}$ depends on the particular decomposition of $\Psi_{t}$. According to Zhang (2007), one obtains the following model:

$$
\begin{gathered}
d S_{t}^{i}=S_{t}^{i}\left(\mu_{t}^{i} d t+\sum_{j=1}^{n} \tilde{\sigma}_{t}^{i j} d \tilde{W}_{t}^{j}\right), \quad i=1, \ldots, n . \\
\theta_{t}=A_{t}^{-1} \cdot \Xi_{t}^{-1} \cdot\left(\mu_{t}-r_{t} 1_{n}\right) \\
\theta_{t}^{2}=\left(\mu_{t}-r_{t} 1_{n}\right)^{T} \cdot\left(\sigma_{t} \sigma_{t}^{T}\right)^{-1}\left(\mu_{t}-r_{t} 1_{n}\right)
\end{gathered}
$$

Completions for the Models with Jumps

The idea of adding auxiliary assets to the market to make it complete is not limited to the diffusion market model. There were also some developments towards a more general geometric Levy model in which asset price is governed by jumps

$$
\begin{aligned}
& d B_{t}=r B_{t} d t \\
& \left.d S_{t}=S_{t-}\left(\mu d t+d Z_{t}\right)\right) \quad S_{0}>0, \\
& Z_{t}=\sigma W_{t}+X_{t}
\end{aligned}
$$

where $X_{t}$ is a pure jump process and $W$ and $X$ are independent variables. It is well known that such Levy model is not complete even in a one-dimensional case as it includes jumps and Brownian motions as two independent sources of risk and only one asset to use. So instead of introducing the same structure auxiliary assets, authors in Corcuera et al. (2005) enlarge the Levy market with the so-called $i$ th-powerjump assets defined as

$$
X_{t}^{(i)}=\sum_{0<s \leq t}\left(\Delta X_{s}\right)^{i}, \quad i \geq 2,
$$

where $\Delta X_{s}=X_{s}-X_{s-}$ and $X_{t}^{(1)}=X_{t}$. Processes $X^{(i)}$ are again Levy processes. These powerjump processes jump at the same time as the original $Z_{t}$; however, jump sizes are the i-th power of jumps of the original process. Note, that $X_{t}^{(i)}=Z_{t}^{(i)}, i \geq 2$. It is convenient to re-write these assets in the compensated form

$$
Y_{t}^{(i)}=Z_{t}^{(i)}-E\left[Z_{t}^{(i)}\right]=Z_{t}^{(i)}-m_{i} t, \quad i \geq 1 .
$$

Enlargement of the model is then consisting in allowing to trade in assets:

$$
H_{t}^{(i)}=e^{r t} Y_{t}^{(i)}, \quad i \geq 2 .
$$

With these assets available, it was demonstrated in Corcuera et al. (2005) that any squareintegrable martingale $M_{t}$ can be represented as follows:

$$
M_{t}=M_{0}+\int_{0}^{t} h_{s} d \tilde{Z}_{s}+\sum_{i=2}^{\infty} h_{s}^{(i)} d Y_{s}^{(i)}
$$

where $h_{s}$ and $h_{s}^{(i)}, i \geq 2$ are predictable processes such that

$$
\tilde{Z}=Z_{t}-(\mu-r) t, t \geq 0
$$

and

$$
\begin{gathered}
E\left[\int_{0}^{t}\left|h_{s}\right|^{2} d s\right]<\infty \\
E\left[\int_{0}^{t}\left|h_{s}^{(i)}\right|^{2} d s\right]<\infty .
\end{gathered}
$$

In other words, for any square-integrable contingent claim $f$ (non-negative, $\mathcal{F}_{T}$ measurable random variable) we can set up a sequence of self-financing portfolios whose final values converge in $L^{2}\left(P^{*}\right)$. This portfolio will consist of a finite number of bonds, stocks and $i$ thpower-jump assets. It means that $f$ can be replicated, and the market is approximately complete.

This interesting result is important to consider within the general idea of market completion because it offers to search for more specific auxiliary assets beyond just structurepreserving ones discussed before. In the case of the Levy market model or another model with jumps, it might be more convenient to pick specific types of completing assets for each kind of risks presented. It is also useful in terms of interpretation of the auxiliary assets as powerjump-assets are by nature instruments that give exposure to moments like variance (2nd- 
power-jump asset) or skewedness and kurtosis of distribution (3rd and 4th correspondingly). Assets of such type might be more convenient to introduce to real markets to fix their incompleteness.

\section{Completions in Optimal Investment and Partial/Imperfect Hedging}

Let us now elaborate more on the application of the method of Market Completions. In this section, we mainly focus on another big part of the area of the Mathematical Finance field hedging of contingent claims with the major focus on partial hedging.

The idea of introducing fictitious assets to complete the market has already demonstrated potential on the side of partial hedging. First, it is reasonable to look at the classical approaches of partial hedging known for complete market and demonstrate potential towards implementing market completions method for the incomplete case. As it is known, the most up-to-date risk measure approved in the Basel III accord is CVaR.

Definition 0.1: Value-at-Risk (VaR) measure of a loss X can be defined as

$$
\operatorname{VaR}_{\alpha}(X)=\inf a: P(X>a) \leq \alpha
$$

Definition 0.2: Conditional Value-at-Risk (CVaR) measure of a loss X can be defined as

$$
\operatorname{CVaR}_{\alpha}(X)=\frac{1}{\alpha} \int_{0}^{\alpha} \operatorname{VaR} R_{\alpha}(x) d x,
$$

The problem of CVaR optimal hedging contingent claim $H$ under budget constraint $x \leq \tilde{V}$ therefore can be stated as

$$
\left\{\begin{array}{c}
\operatorname{CVaR}_{\alpha}(x, \pi) \rightarrow \min _{(x, \pi)} \\
x \leq \tilde{V}
\end{array}\right.
$$

Inspired by Rockafellar and Urasev, Melnikov and Smirnov in Melnikov and Smirnov (2012) demonstrated that, introducing the special function of parameter $z \in \mathbb{R}$

$$
c(z)=z+\frac{1}{1-\alpha} \min _{(x, \pi)} \mathbb{E}\left[\left(H(z)-V_{T}^{\pi}(x)\right)^{+}\right]
$$

where $H(z)=(H-z)^{+}-$modified contingent claim $H$, the problem of CVaR minimisation in case of European contingent claim will be equivalent to the following one:

$$
\min _{z \in \mathbb{R}} c(z)=\min _{(x, \pi)} C V a R_{\alpha}(x, \pi) .
$$

Consequently, solution of (16) can be decomposed into consequent optimisation by $z$ after solving “internal” problem:

$$
\left\{\begin{array}{c}
E\left[\left(\mathrm{H}(\mathrm{z})-\mathrm{V}_{\mathrm{T}}^{\mathrm{p}}(\mathrm{x})\right)^{+}\right] \\
x \leq \tilde{V}
\end{array}\right.
$$

Alternatively, one can approach this problem from the perspective of optimal split into hedged/unhedged proportions of the claim $H=f(H)+R_{f}(H)$, where $f(H)$ describes the optimal hedged proportion of the claim. This method was offered by Cong et al. (2014).

Considering European type contingent claim, we expect to have a pay-off at maturity time $T$, so the total risk exposure of the investor is going to be

$$
T_{f}(X)=R_{f}(X)+e^{r T} \Pi(f(X)),
$$

where $\Pi(f(X))$ is some chosen pricing functional for the hedged part of exposure.

Given the initial budget constraint, the investor is pursuing the goal of minimising risk measure of total exposure (18), given the restriction on the initial cost of hedging

$$
\left\{\begin{array}{l}
\min _{f \in \Omega} \operatorname{CVaR}\left(T_{f}(X)\right) \\
\text { s.t. } \Pi(f(X)) \leq \pi_{0}
\end{array}\right.
$$

According to Cong et al. (2014), under particular assumptions, an explicit way of identifying the optimal hedged loss function is stated in the following theorem.

Theorem 4: Assume that pricing functional is linear for any time- $\mathrm{t}$ contingent payout $\mathrm{Z}$. Then, the optimal hedged loss function $\mathrm{g}_{\mathrm{f}}^{*}$ is given by

$$
g_{f}^{*}(x)=\left(x-d^{*}\right)^{+}-\left(x-u^{*}\right)^{+}
$$

where $\left(d^{*}, u^{*}\right)$ satisfies the following equations 


$$
\left\{\begin{array}{l}
e^{-r T} \int_{d^{*}}^{u^{*}} \mathbb{Q}(X>x) d x=\pi_{0} \\
\mathbb{P}\left(X>u^{*}\right)=\alpha \cdot \frac{\mathbb{Q}\left(X>u^{*}\right)}{\mathbb{Q}\left(X>d^{*}\right)}
\end{array}\right.
$$

and $\mathbb{Q}$ is a risk-neutral measure.

In both approaches, we arrive at some known problem that is well described for the complete market. Consequently, completion of the market can be helpful as it helps to "parametrise" a solution by the set of completing assets. Therefore, choice of the proper completion by market conditions such as partial equilibrium $\mathrm{Hu}$ et al. (2005), Esscher measure or Minimal Relative Entropy Measure will be a powerful tool for solving CVaR optimisation problems on the incomplete market.

To demonstrate sensibility of usage of Method of Market Completions for solving stated partial hedging problem (17), we provide existing techniques of partial hedging where Method of Market Completions has already demonstrated great potential or ready to be implemented.

\section{Utility Maximisation}

Let us start with the simple case when the goal of the investor is to finance a strategy that provides the greatest terminal wealth utility.

$$
v_{0}(x) \equiv \sup _{\pi \in \mathcal{A}(x)} E\left[U\left(V_{T}^{\pi}(x)\right)\right] .
$$

In Karatzas et al. (1991), authors have shown how to obtain such optimal solution with the help of convex duality methods. In the core of these methods lies Legendre-Fenchel transform $\tilde{U}(y) \equiv \max _{x>0}(U(x)-x y)=U(I(y))-y I(y)$, where

$I: \mathbb{R}^{+} \rightarrow \mathbb{R}^{+}$is defined as the continuous decreasing inverse function of $U^{\prime}(x)$ (details in Touchette, n.d.).

The solution to this problem for the complete market was given explicitly and can be summarised as the following theorem:

Theorem 5: For a given initial budget $\tilde{\mathrm{V}}_{0}>0$, under the assumption that function

$$
\mathcal{X}_{0}(y) \equiv E\left[\beta_{T} Z_{T}^{0} \cdot I\left(y \beta_{T} Z_{T}^{0}\right)\right]\langle\infty, \forall y\rangle 0,
$$

the optimal terminal wealth of a strategy can be found as

$$
\xi_{0}^{\widetilde{V}_{0}}=I\left(\mathcal{Y}_{0}\left(\tilde{V}_{0}\right) \beta_{T} Z_{T}^{0}\right)
$$

where $\mathcal{Y}_{0}$ is the inverse of the function $\mathcal{X}_{0}$.

By introducing martingale $X_{t} \equiv E\left[\beta_{T} Z_{T}^{0} \xi_{0}^{x} \mid \mathcal{F}_{t}\right]$ with stochastic integral representation $X_{t}=\tilde{V}_{0}+\int_{0}^{t} \varphi_{s}^{T} d W_{s}$ with $\varphi \in \mathcal{F}_{t}$ and $\int_{0}^{T}\left\|\varphi_{s}\right\|^{2} d s<\infty$, replicating portfolio for optimal terminal capital can be obtained as

$$
\hat{\pi} \equiv \frac{1}{X_{t}}\left(\Sigma_{t}^{T}\right)^{-1}\left(\varphi_{t}+X_{t} \theta_{t}\right) .
$$

Applying the Method of Market Completions for the case of incomplete markets, one can introduce $k-n$ fictitious assets in addition to $n$

existing assets on the incomplete market, driven by the same $k$-dimensional Brownian motion as $n$ real tradeable assets. Then, the problem of utility maximisation can be solved in the completed market with fictitious assets, but there are infinitely many ways to introduce those fictitious assets.

The relative risk process can then be represented as $\tilde{\theta}_{t} \equiv \theta_{t}+v_{t}$ with $\theta_{t}^{T} v_{t}=0$. That means completions could be parametrised by $v$ which is square-integrable, $\mathcal{F}_{t}$ adapted and $\mathbb{R}^{d}$ valued process.

Denote also exponential local martingale:

$$
Z_{t}^{v} \equiv \exp \left\{-\int_{0}^{t} \tilde{\theta}_{s}^{T} d W_{s}-\frac{1}{2} \int_{0}^{t}\left(\theta_{s}{ }^{2}+v_{s}{ }^{2}\right) d s\right\}
$$

and the function

$$
\forall y>0, \quad \mathcal{X}_{\mathrm{v}}(y) \equiv E\left[\beta_{T} Z_{T}^{v} I\left(y \beta_{T} Z_{T}^{v}\right)\right] .
$$

Also $\forall v \in K_{1}(\Sigma)$ where

$$
\begin{aligned}
& K_{1}(\Sigma) \equiv v \in K(\Sigma), \mathcal{X}_{v}(y)\langle\infty, \forall y\rangle 0, \text { define } \\
& \xi_{v}^{x} \equiv I\left(\mathcal{Y}_{v}(x) \beta_{T} Z_{T}^{v}\right)
\end{aligned}
$$

where $\mathcal{Y}_{v}$ again is the inverse function of $\mathcal{X}_{v}$

An attainable solution will give us value less or equal than that. If we find a strategy $\pi$ with initial capital $x$, which does not require the purchase of the artificial stocks and completion $\lambda_{t} \in K_{1}(\Sigma)$ such that 


$$
\sup _{\pi \in \mathcal{A}(x)} E\left[U\left(V_{T}^{\pi}\right)\right]=E\left[U\left(\xi_{\lambda}^{x}\right)\right],
$$

then, for sure, $(\pi, \lambda)$ would be optimal. In Karatzas et al. (1991) the following theorem was proven.

Theorem 6: If we call
Optimality of $\pi: E U\left(V_{T}^{\pi}\right) \leq E U\left(V_{T}^{\hat{\pi}}\right) \quad \forall \pi \in \mathcal{A}(x)$

Financiability of $\xi_{\lambda}^{x}: \exists \hat{\pi} \in \mathcal{A}(x)$ such that $V_{T}^{\hat{\pi}}=\xi_{\lambda}^{x}$

Least Favorability of $\lambda$ :

$$
E U\left(\xi_{\lambda}^{x}\right) \leq E U\left(\xi_{v}^{x}\right) \quad \forall v \in K_{1}(\Sigma)
$$

Parsimony of $\lambda: E\left[\beta_{T} Z_{T}^{v} \xi_{\lambda}^{x}\right] \leq x, \quad \forall v \in K_{1}(\Sigma)$

Then $B \Leftrightarrow D \Rightarrow C$.

Furthermore, if $B$ holds, then the portfolio $\hat{\pi}$ in B satisfies $A$.

This theorem provides a powerful instrument in verifying if one can build an optimal strategy without artificial assets in use, in other words, when $\lambda=0$ will satisfy necessary criteria in Theorem 1. It was shown to be the case in Karatzas et al. (1991) for $U(X)=\ln (X)$ and, under some special conditions for $U(X)=\frac{X^{\delta}}{\delta}$ where $\delta<1, \delta \neq 0$. This edge can further be applied to more specific problems of partial hedging. We provide one example from Karatzas et al. (1991) to demonstrate the application of these conditions to the classical logarithm utility function.

Example 1: Classical example of Utility function to consider is $\mathrm{U}(\mathrm{x})=\ln (\mathrm{x})$. For this function one has:

$$
\mathcal{X}_{v}(y)=\frac{1}{y}, \quad \mathcal{Y}_{v}(x)=\frac{1}{x}
$$

and optimal terminal capital can be calculated as

$$
\xi_{v}^{x}=\frac{x}{\beta_{T} Z_{T}^{v}} .
$$

One could check that completion with parameter $\lambda=0$ satisfies $D$.

$$
E\left[\beta_{T} Z_{T}^{v} \xi_{0}^{x}\right]=x \cdot E\left[\exp \left\{\begin{array}{c}
-\int_{0}^{T} v_{s}^{T} d W_{s}- \\
-\frac{1}{2} \int_{0}^{T}\left\|v_{s}\right\|^{2} d s
\end{array}\right\}\right] \leq x \quad \forall v \in K(\Sigma)
$$

as the process under expectation is a supermartingale. It means that investor would not use auxiliary stocks to form an optimal portfolio even for hedging purposes.

\section{Efficient Hedging}

One of such problems emerges when given an amount of initial capital $v_{0}$ investor's goal is to find the admissible strategy with terminal wealth $V_{T}$ such that

$$
\left\{\begin{array}{l}
\mathbb{E}\left[U\left(\left(H-V_{T}\right)^{+}\right)\right]=\min \\
\sup _{P^{*} \in \mathcal{P}} \mathbb{E}^{*}\left[V_{T}\right] \leq v_{0}
\end{array}\right.
$$

Föllmer and Leukert demonstrated in Föllmer and Leukert (2000) that such problem can also be solved with the help of convex duality methods, similar to utility maximisation, as one can define state-dependent utility function

$$
u(x, \omega)=U(H(\omega))-U\left((H(\omega)-x)^{+}\right) .
$$

And then re-write (19) in the following form

$$
\left\{\begin{array}{l}
\mathbb{E}\left[u\left(V_{T}, \omega\right)\right]=\max \\
\sup _{P^{*} \in \mathcal{P}} \mathbb{E}^{*}\left[V_{T}\right] \leq v_{0}
\end{array}\right.
$$

which can be solved explicitly on the complete market.

For each $z \leq E^{*}[H]$ there is a unique terminal wealth $\tilde{Z}$ such that

$$
E[U(\tilde{Z}, .)]=\sup \left\{E[U(Z, .)] \mid 0 \leq Z \leq H, E^{*}[Z] \leq z\right\} .
$$

It takes the form

$$
\tilde{Z}=I\left(y(z) Z_{T}^{0}(\omega), \omega\right) \wedge H(\omega)
$$

where $y(z)$ is the solution of

$$
E^{*}\left[I\left(y(z) Z_{T}^{0}(\omega), \omega\right) \wedge H(\omega)\right]=z
$$

Obviously, such a reduction provides strong evidence that one can move on in the direction of Theorem 1 to elaborate on mentioned criteria and generalise them for this category of problems. 


\section{Quantile Hedging}

An important case of efficient hedging is when we focus on minimising the expected size of the shortfall, or $U(X)=X$. This particular case is extremely useful for solving (17) to find the solution of CVaR optimisation problem (16).

Apart from applying similar convex duality methods Spivak and Cvitanic (1999), one can use an alternative approach, which involves the famous Neyman-Pearson lemma. According to Föllmer and Leukert (1999), it is enough to solve the equivalent problem

$$
\left\{\begin{array}{c}
\int \varphi d Q \rightarrow \max \\
\int \varphi d Q^{*} \leq \frac{\tilde{V}_{0}}{E^{*}[H]}, \forall P^{*} \in P
\end{array}\right.
$$

where

$$
\frac{d Q}{d P}=\frac{H}{E[H]}, \quad \frac{d Q^{*}}{d P^{*}}=\frac{H}{E^{*}[H]} .
$$

The solution to problems of such a type was demonstrated in Föllmer and Leukert (1999) and can be found as a perfect hedge for a modified claim $\tilde{H}=H \tilde{\varphi}$ where

$$
\begin{gathered}
\tilde{\varphi}=I_{\frac{d P}{d P^{*}}}+\tilde{\gamma} I_{\frac{d P}{d P^{*}}=\tilde{a}} \\
\tilde{a}=\inf \left(a \geq 0 \mid E^{*}\left[H I_{\frac{d P}{d P^{*}}>\tilde{a}}\right] \leq \tilde{V}\right) \\
\tilde{\gamma}=\frac{\tilde{V}-E^{*}\left[H I_{\frac{d P}{d P^{*}}>\tilde{a}}\right]}{E^{*}\left[H I_{\frac{d P}{d P^{*}}=\tilde{a}}\right]}
\end{gathered}
$$

It is easy to notice that the solution is based on finding maximal successful hedging set, which can be represented as $\left\{\frac{d P}{d P^{*}}>\right.$ Const $\left.\times H\right\}$, where

$H$ is some claim. With the reasonable assumption that claim $H$ depends on some existing asset $S_{T}^{i}$ and using the following representation on the complete market

$$
\frac{d P_{T}}{d P_{T}^{*}}=\exp \left\{\theta^{T} \tilde{W}_{T}-\frac{1}{2} \theta^{2} T\right\}=\left(S_{T}^{i}\right)^{1 / \varphi^{i}} \times \Lambda^{i}
$$

where $\varphi^{i}=\frac{\mu^{i}-r}{\|\theta\|^{2}}$, successful hedging set can be found in the form of

$$
\left\{\left(S_{T}^{i}\right)^{\frac{1}{\varphi^{i}}} \times \Lambda^{i}>\text { Const } \times H\left(S_{T}^{i}\right)\right\}
$$

which, in the case of one dimension, coincides with the solution described in Melnikov et al. (2001).

In an incomplete market case, we again add some auxiliary assets into consideration. As was demonstrated above, one can develop innovative Brownian Motion, under which, last $(k-n)$ coefficients of each row $\sigma^{i}$ for existing assets in the "completed" volatility matrix will be equal 0 . Then, using representation (6), if claim $H$ still depends on existing assets only, it is possible to show that

$$
\left\{\frac{d P_{T}}{d P_{T}^{*}}>a \cdot H\right\}=\left\{Z_{\text {asset }}^{-1} \cdot Z_{\text {completion }}^{-1}>a \cdot H(\text { asset })\right\}
$$

Consequently, it is reasonable to develop a general theory of applying Method of Market Completions to the construction of a successful hedging set. It helps reduce the Quantile Hedging problem to operations with existing assets only.

\section{Conclusion}

In this paper Method of Market Completions is introduced as a dual approach for operating on incomplete markets. It was demonstrated that in the case of pricing problem, this approach leads to the same solution as classical ones. As the method of market completions offers an alternative way of working with standard problems of mathematical finance in incomplete markets, it was shown how to reduce such problems to the known version in the complete market.

In line with it, alternative ways of handling market incompleteness were observed with their connection to the method of market completions and possible future developments and improvements of the presented method. Further enhancements of this method consist in finding 
a way for reverse-engineering parameters of the completion required utilising BSDE, partial equilibrium market condition or using another asset class like bonds or insurance contracts. On the other hand, it will also be beneficial to find a way of choosing the most suitable completion according to market conditions and investors goals.

\section{Acknowledgements}

The research was supported by the NSERC Discovery Grant RES 0043487.

\section{References}

Bajeux-Besnainou, I., \& Portait, R. (1997). The numeraire portfolio: a new perspective on financial theory. The European Journal of Finance, 3(4), 291-309. https://EconPapers.repec.org/RePEc: taf: eurjfi: v:3: y:1997: i:4: p:291-309

Capinski, M. (2014). Hedging Conditional Value at Risk with Options. European Journal of Operational Research. https://doi.org/10.1016/j.ejor.2014.11.011

Cong, J., Tan, K. S., \& Weng, C. (2014). Conditional value-at-risk-based optimal partial hedging. The Journal of Risk, 16(3), 49-83.

Corcuera, J. M., Nualart, D., \& Schoutens, W. (2005). Completion of a Lévy market by power-jump assets. Finance and Stochastics, 9(1), 109.

Dhaene, J., Kukush, A., \& Linders, D. (2013). The Multivariate Black-Scholes Market: Conditions for Completeness and No-Arbitrage. Theory of Probability and Mathematical Statistics, 88, 1-14. https://doi.org/10.2139/ ssrn. 2186830

Eyraud-Loisel, A. (2019). How Does Asymmetric Information Create Market Incompleteness? Methodology and Computing in Applied Probability, 21(2). https://doi.org/10.1007/s11009-018-9672-x

Föllmer, H., \& Leukert, P. (1999). Quantile hedging. Finance and Stochastics, 3(3), 251-273. https://EconPapers. repec.org/RePEc: spr: finsto: v:3: y:1999: i:3: p:251-273

Föllmer, H., \& Leukert, P. (2000). Efficient hedging: Cost versus shortfall risk. Finance and Stochastics, 4, 117-146.

Follmer, H., \& Schweizer, M. (1991). Hedging of Contingent Claims Under Incomplete Information. (389-414). In M.H.A. Davis \& R. J. Elliott (eds.), Applied Stochastic Analysis, Stochastics Monographs, Vol. 5, Gordon and Breach, London/New York.

Godin, F. (2015). Minimising CVaR in global dynamic hedging with transaction costs. Quantitative Finance, 16, 1-15. https://doi.org/10.1080/14697688.2015.1054865

Guilan, W. (1999). Pricing and hedging of American contingent claims in incomplete markets. Acta Mathematicae Applicatae Sinica, 15, 144-152. https://doi.org/10.1007/BF02720489

Hu, Y., Imkeller, P., \& Müller, M. (2005). Partial Equilibrium and Market Completion. International Journal of Theoretical and Applied Finance (IJTAF), 08, 483-508. https://doi.org/10.1142/S 0219024905003098

Karatzas, I., Lehoczky, J. P., Shreve, S. E., \& Xu, G.-L. (1991). Martingale and Duality Methods for Utility Maximization in an Incomplete Market. SIAM J. Control Optim., 29(3), 702-730. https://doi.org/10.1137/0329039

Karatzas, I., \& Shreve, S. (2000). Methods of Mathematical Finance. Journal of the American Statistical Association, 95. https://doi.org/10.2307/2669426

Kobylanski, M. (2000). Backward Stochastic Differential Equations and Partial Differential Equations with Quadratic Growth. The Annals of Probability, 28(2), 558-602.

Li, J., \& Xu, M. (2013). Optimal Dynamic Portfolio with Mean-CVaR Criterion. Risks, ISSN 2227-9091, MDPI, Basel, Vol. 1, Iss. 3, pp. 119-147, http://dx.doi.org/10.3390/risks1030119

Melnikov, A. (1999). Financial Markets: Stochastic Analysis and the Pricing of Derivative Securities. American Mathematical Society.

Melnikov, A., \& Smirnov, I. (2012). Dynamic hedging of conditional value-at-risk. Insurance: Mathematics and Economics, 51. https://doi.org/10.1016/j.insmatheco.2012.03.011

Melnikov, A., Volkov, S., \& Nechaev, M. (2001). Mathematics of Financial Obligations.

Melnikov, A. V., \& Feoktistov, K. M. (2001). Вопросы безарбитражности и полноты дискретных рынков и расчеты платежных обязательств [Arbitration-Free and Completeness Issues for Discrete Markets and Calculations of Payment Obligations]. Obozreniye Prikladnoy i Promyshlennoy Matematiki, 8(1), 28-40. (In Russian) 
Miyahara, Y. (1995). Canonical Martingale Measures of Incomplete Assets Markets. Probability Theory and Mathematical Statistics: Proceedings of the Seventh Japan-Russia Symposium.

Spivak, G., \& Cvitanic, J. (1999). Maximising the probability of a perfect hedge. The Annals of Applied Probability, 9(4), 1303-1328

Touchette, H. (n.d.). Legendre-Fenchel transforms in a nutshell. Retrieved from https://www.ise.ncsu.edu/fuzzyneural/wp-content/uploads/sites/9/2019/01/or706-LF-transform-1.pdf

Zhang, A. (2007). A secret to create a complete market from an incomplete market. Applied Mathematics and Computation, 191, 253-262. https://doi.org/10.1016/j.amc.2007.02.086

\section{ABOUT THE AUTHORS / ИНФОРМАЦИЯ ОБ АВТОРАХ}

Ilia Vasilev - PhD Candidate; MSc Moscow Institute of Physics and Technology (State University); BSc Moscow Institute of Physics and Technology (State University). University of Alberta, Edmonton, AB, Canada Илья Васильев - аспирант; получал степень бакалавра и магистра в Государственном институте физики и технологии. Университет Альберты, Эдмонтон, АВ, Канада. ivasilev@ualberta.ca

Alexander Melnikov - Professor, DSc from Steklov Mathematical Institute, Russia; PhD from Steklov Mathematical Institute, Russia; MSc from Moscow State University, Russia. University of Alberta, Edmonton, AB, Canada

Александр Мельников - профессор, доктор физико-математических наук. Степень кандидата и доктора наук получил в Математическом институте им. В.А. Стеклова РАН. Выпускник Московского государственного университета им. М.В. Ломоносова. Университет Альберты, Эдмонтон, АВ, Канада. melnikov@ualberta.ca

The article was submitted on 17.05.2021, reviewed on 16.07.2021, and accepted for publication on 17.08.2021. The authors read and approved the final version of the manuscript.

Статья поступила в редакцию 17.05.2021; после рецензирования 16.07.2021; принята к публикации 17.08.2021.

Авторы прочитали и одобрили окончательный вариант рукописи. 